\title{
Hierarchically Clustered Star Formation in the Magellanic Clouds
}

\author{
Dimitrios A. Gouliermis, Stefan Schmeja, Volker Ossenkopf, Ralf S. Klessen, and \\ Andrew E. Dolphin
}

\begin{abstract}
We present a cluster analysis of the bright main-sequence and faint premain-sequence stellar populations of a field $\sim 90 \times 90 \mathrm{pc}$ centered on the HII region NGC 346/N66 in the Small Magellanic Cloud, from imaging with HST/ACS. We extend our earlier analysis on the stellar cluster population in the region to characterize the structuring behavior of young stars in the region as a whole with the use of stellar density maps interpreted through techniques designed for the study of the ISM structuring. In particular, we demonstrate with Cartwrigth \& Whitworth's $\mathscr{Q}$ parameter, dendrograms, and the $\Delta$-variance wavelet transform technique that the young stellar populations in the region NGC 346/N66 are highly hierarchically clustered, in agreement with other regions in the Magellanic Clouds observed with HST. The origin of this hierarchy is currently under investigation.
\end{abstract}

\section{Method: The identification of stellar clusters}

For the investigation of the clustering behavior of stars it is necessary to thoroughly characterize distinct concentrations of stars, which can only be achieved by the ac-

Dimitrios A. Gouliermis · Ralf S. Klessen

Zentrum für Astronomie der Universität Heidelberg, Institut für Theoretische Astrophysik, Albert-Ueberle-Str. 2, 69120 Heidelberg, Germany

e-mail: dgoulier@mpia-hd.mpg.de, klessen@uni-heidelberg.de

Stefan Schmeja

Zentrum für Astronomie der Universität Heidelberg, Astronomisches Rechen-Institut, Mönchhofstr. 12-14, 69120 Heidelberg, Germany e-mail: sschmeja@ ari.uni-heidelberg.de

Volker Ossenkopf

I. Physikalisches Institut der Universität zu Köln, Zülpicher Straße 77, 50937 Köln, Germany e-mail: ossk@ph1.uni-koeln.de

Andrew E. Dolphin

Raytheon Company, PO Box 11337, Tucson, AZ 85734, USA e-mail: adolphin@raytheon.com 

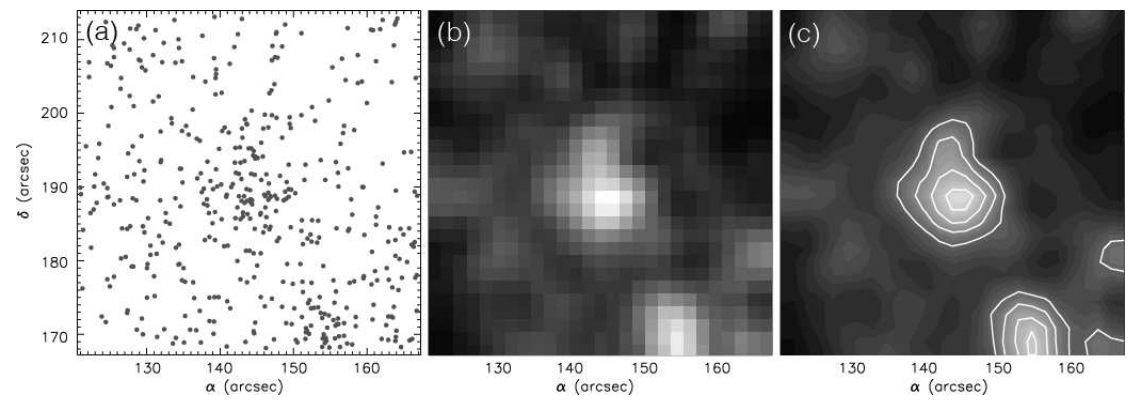

Fig. 1 Schematic of the star-count process. (a) The chart of an observed stellar sample. (b) The corresponding stellar density map, after counting stars in quadrilateral grid of elements (pixels) of size $1.8^{\prime \prime}$ each, and after filtering the map with a Gaussian of FWHM $\simeq 2.8 \mathrm{px}\left(\sim 5^{\prime \prime}\right)$. (c) The corresponding isodensity contour map. Isopleths at levels $\gtrsim 3 \sigma$ are indicated with white lines.

curate identification of individual stellar clusters. Considering the importance of this process, different identification methods were developed, which can be classified in two families. The first, represented by friend of friend algorithms and cluster analysis techniques, e.g., [2], are designed for limited samples of observed stars, and thus are based on linking individual stars into coherent stellar groups. These methods are recently superseded by minimum spanning trees, e.g., [1]. The second family of identification codes, represented by nearest-neighbors and star-counts, make use of surface stellar density maps constructed from rich observed stellar samples. Distinct stellar systems are identified as statistically significant over-densities in respect to the average stellar density in the observed regions, e.g., [5]. Tests on artificial clusters of various density gradients and shapes showed that the latter (density) techniques are more robust in detecting real stellar concentrations, provided that rich stellar samples are available [10]. A schematic representation of stellar density maps constructed with star-counts is shown in Fig. 1.

\section{Data: Stellar clustering in the region NGC 346/N66}

One of the most prominent bright stellar systems in the Small Magellanic Cloud (SMC) is the stellar association NGC 346, related to the HII region LHA 115-N66 [6], the brightest in this galaxy. This system appears in partially-resolved observations form the ground as a single stellar concentration, but recent imaging with the Advanced Camera for Surveys onboard the Hubble Space Telescope (HST) allowed the detection of smaller sub-clusters within the boundaries of the HII nebula. The images were collected within the HST GO Program 10248 and were retrieved from the HST Data Archive. Their photometry demonstrated that the faint young stellar populations in the region are still in their pre-main-sequence (PMS) phase, and revealed a plethora of sub-solar PMS stars [4]. Our nearest-neighbor cluster analysis of the observed young stellar populations, i.e., the bright main-sequence (down to 
Fig. 2 Isodensity contour map from star-counts of the young bright main-sequence and faint PMS populations identified with HST/ACS in the region of NGC 346 in the SMC. Lines represent isopleths of significance $\gtrsim 1 \sigma$. Apart from the dominating central large stellar aggregate, there are peripheral young sub-clusters, revealed as statistically important stellar concentrations. The central aggregate, denoted by the $1 \sigma$ isopleth, encompass various distinct sub-groups, which appear at higher density thresholds. NGC 346 itself appears at $\gtrsim 3 \sigma$ significance.

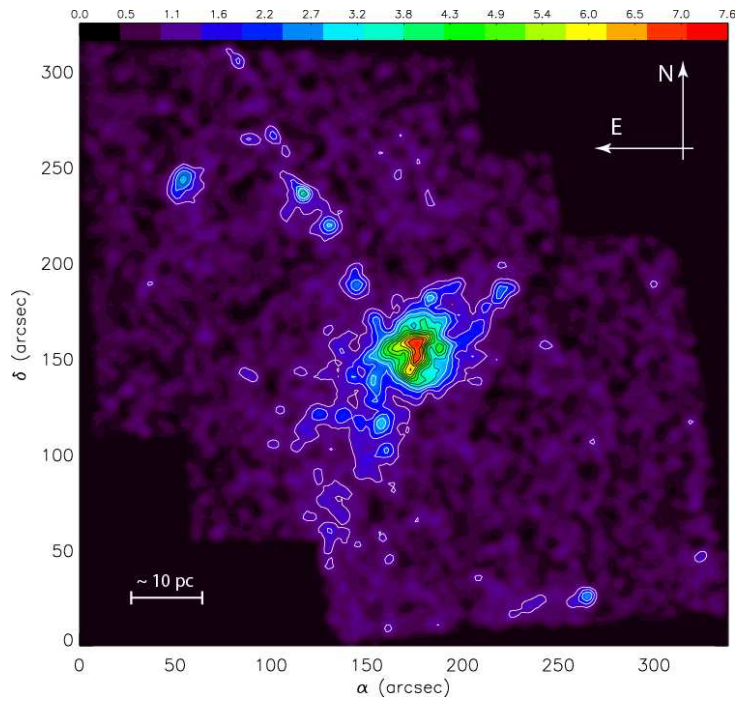

$\left.m_{555} \lesssim 21\right)$ and the faint PMS stars, revealed a significant number of smaller, previously unresolved, young stellar sub-clusters [9]. This clustering behavior of young stars in NGC 346 is further demonstrated here by the stellar density contour map of Fig 2, constructed with star-counts.

\section{Results: Hierarchical clustering of young stars}

The map of Fig. 2 shows significant sub-structure, in particular within the $1 \sigma$ boundaries of the central dominant stellar aggregate. This structuring behavior indicates hierarchy. The minimum spanning tree (MST) of the young stars in the whole region allows to determine the statistical $\mathscr{Q}$ parameter, introduced by [3]. This parameter is a measure of the fractal dimension $D$ of a stellar group, permitting to distinguish between centrally concentrated clusters and hierarchical clusters with fractal substructure. The application of the MST to our data shows that the region NGC 346/N66 is highly hierarchical with a $\mathscr{Q}$ that corresponds to a fractal dimension $D \simeq 2.5$.

Constructing surface stellar density maps allows us to further characterize the clustering behavior of stars with the application of tools, which are originally designed for the study of the structuring of the interstellar medium (ISM), as observed at far-infrared or longer wavelengths. The so-called dendrograms are used for the visualization of hierarchy through structural trees [8]. The dendrogram of the stellar density map of NGC 346 demonstrates that the observed hierarchy is mostly due to the substructure in the dominant stellar aggregate. The $\Delta$-variance analysis $[11,7]$ is a robust structure analysis method that measures the amount of structure on a given scale $l$. In principle the $\Delta$-variance is directly related to the power spectrum of 
Fig. 3 The $\Delta$-variance spectrum of the surface stellar density map of the entire region of NGC 346/N66. This analysis shows that the young stellar populations in this region are hierarchically structured up to length-scales of $\sim 40^{\prime \prime}$. The spectral index $\beta$ is determined from the fit of the spectrum for data between lags $4^{\prime \prime}$ and $13^{\prime \prime}$ (indicated by the gray shaded area). The dashed line provides the used virtual beamsize $\left(5^{\prime \prime}\right)$.

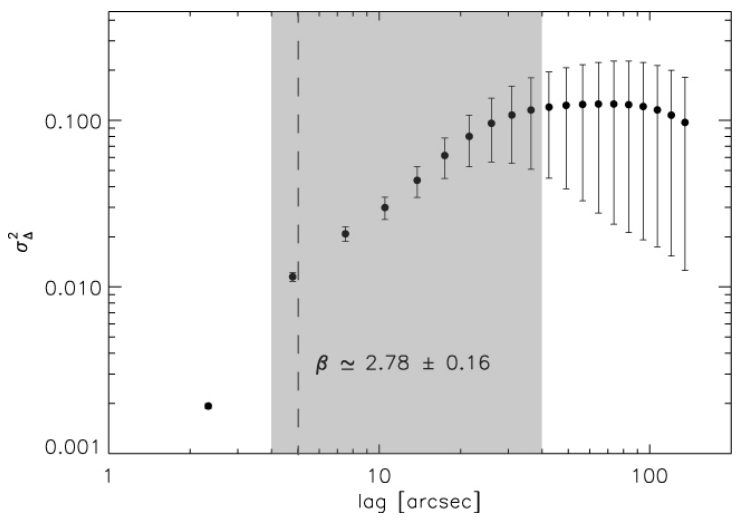

the map, and thus for a power law spectrum of index $-\beta, \Delta$-variance also follows a power law, $\sigma_{\Delta}^{2} \propto l^{\alpha}$, with $\alpha=\beta-2$. The application of the $\Delta$-variance analysis on the surface stellar density map of NGC 346 verifies that indeed the clustering of the young stars in the region is self-similar (Fig. 3), with a spectral index $\beta \simeq 2.8$, corresponding to a fractal dimension $D=2.6$ of the corresponding fractional Brownian motion structure [11], similar to that previously derived for Galactic molecular clouds. Self-similarity appears to brake, i.e., we find different hierarchical properties for the short-range scaling and the behavior at the overall scale of the region, at length-scales $l \geq 25 \mathrm{px}$, corresponding to physical scales of $\sim 40^{\prime \prime}(\sim 11 \mathrm{pc}$ at the distance of the SMC).

Acknowledgements D.A.G., S.S. and V.O. kindly acknowledge support by the German Research Foundation (DFG) through grants GO 1659/3-1, SFB 881 and OS 177/2-1 respectively. Based on observations made with the NASA/ESA Hubble Space Telescope, obtained from the data archive at the Space Telescope Science Institute (STScI). STScI is operated by the Association of Universities for Research in Astronomy, Inc. under NASA contract NAS 5-26555.

\section{References}

1. Bastian, N., et al. 2009. Mon. Not. R. Astron. Soc. 392, 868

2. Battinelli, P., Efremov, Y., \& Magnier, E. A. 1996. Astron. Astrophys. 314, 51

3. Cartwright, A., \& Whitworth, A. P. 2004. Mon. Not. R. Astron. Soc. 348, 589

4. Gouliermis, D. A., et al. 2006. Astroph. J. Suppl. Ser. 166, 549

5. Gouliermis, D. A., et al. 2010. Astroph. J. 725, 1717

6. Henize, K. G. 1956. Astroph. J. Suppl. Ser. 2, 315

7. Ossenkopf, V., Krips, M., \& Stutzki, J. 2008. Astron. Astrophys. 485, 917

8. Rosolowsky, E. W., et al. 2008. Astroph. J. 679, 1338

9. Schmeja, S., Gouliermis, D. A., \& Klessen, R. S. 2009. Astroph. J. 694, 367

10. Schmeja, S. 2011, Astronomische Nachrichten, 332, 172

11. Stutzki J., et al. 1998. Astron. Astrophys. 336, 697 